\title{
A multi-objective location routing problem using imperialist competitive algorithm
}

\author{
Amir Mohammad Golmohammadi ${ }^{a^{*}}$, Shahrokh Amanpour Bonab ${ }^{\mathrm{b}}$ and Amir Parishani ${ }^{\mathrm{c}}$
}

${ }^{a}$ Ph.D Student of Industrial Engineering,University of Yazd, Yazd, Iran

${ }^{b}$ Young Researchers and Elite Club, Central Tehran Branch, Islamic Azad University, Tehran, Iran

${ }^{c}$ Department of Industrial Engineering, Naragh Branch, Islamic Azad University, Naragh, Iran

\section{H R O N I C L E \\ A B S T R A C T}

Article history:

Received August 312015

Received in Revised Format

Septmber 262015

Accepted December 22015

Available online

December 72015

Keywords:

Optimization

Mathematical Programming

Locating storage unit

Vehicle routing

The meta-heuristic Algorithm

\begin{abstract}
Nowadays, most manufacturing units try to locate their requirements and the depot vehicle routing in order to transport the goods at optimum cost. Needless to mention that the locations of the required warehouses influence on the performance of vehicle routing. In this paper, a mathematical programming model to optimize the storage location and vehicle routing are presented. The first objective function of the model minimizes the total cost associated with the transportation and storage, and the second objective function minimizes the difference distance traveled by vehicles. The study uses Imperialist Competitive Algorithm (ICA) to solve the resulted problems in different sizes. The preliminary results have indicated that the proposed study has performed better than NSGA-II and PAES methods in terms of Quality metric and Spacing metric.
\end{abstract}

(C) 2016 Growing Science Ltd. All rights reserved

\section{Introduction}

Management teams of production and industrial units always try to find a proper location and optimal transportation distribution depots to reduce their production and distribution costs. By looking at both urban distribution depots vehicle routing and facilities for inventories we may find an optimum solutions for both problems, simultaneously. Webb (1968) and Christofides and Eilon (1969) are believed to be the first who explained that it would not be a good idea to consider distribution cost as part of transportation in facility location problems. In competitive environment, customers prefer to receive the products and services at lower cost. According to Christofides and Eilon (1969), the vehicle-scheduling problem includes the design of different vehicle tours to reach a given set of necessities for customers with known locations, subject to a capacity constraint for the vehicles and a distance/time constraint for vehicle tours. Most wholesale distribution is excuted during multiple-delivery journeys. Many techniques of locating depots utilize simple functions of delivery data such as weight and distance from the depot, to measure the delivery "cost"; the total "cost" is minimized to deternine the depot location (Webb, 1968). Tavakkoli-Moghaddam et al.

* Corresponding author.

E-mail: Amir.m.golmohammadi@stu.yazd.ac.ir (A. M. Golmohammadi)

(C) 2016 Growing Science Ltd. All rights reserved.

doi: $10.5267 /$ j. ijiec.2015.12.002 
(2006) considered a vehicle routing problem with backhauls (VRPB) where a set of costumers are categorized in two subsets consisting of linehaul and backhaul costumers. They used memetic algorithm to solve the resulted VRPB. Arvan et al. (2015) designed a bi-objective and multi-product supply chain network for the supply of blood. Teimuory et al. (2013) devloped a multi-objective reliable programming model for disruption in supply chain.

This paper presents a mathematical programming model to optimize the storage location and vehicle routing. The first objective function of the model minimizes the total cost associated with the transportation and storage of rental fee, and the second objective function minimizes the difference distance traveled by vehicles.

\section{The proposed study}

We first introduce the mathematical model by introducing sets, parameters and variables, etc.

Sets

I Set of all distribution centers

$J \quad$ Set of all customers

$K \quad$ Set of all transportation facilities

\section{Parameters}

\begin{tabular}{|c|c|c|c|}
\hline$N$ & Number of customers & $d_{j}$ & Demand for each customer $j$ \\
\hline$D_{i j}$ & Distance between customer $i$ and $j$ & $s_{j k}$ & $\begin{array}{l}\text { Vehicle service time of customer } j \text { by } \\
\text { transportation } k\end{array}$ \\
\hline$C_{i j}$ & Unit cost of travelling between $i$ and $j$ & $w_{j k}$ & $\begin{array}{l}\text { Vehicle waiting time of customer } j \text { by } \\
\text { transportation } k\end{array}$ \\
\hline$t_{i j}$ & Unit time of travelling between $i$ and $j$ & $Q$ & Fixed capacity of transportation vehicle \\
\hline & Fixed cost of establishing distribution center $i$ & $B$ & Total traveling time spent by vehicle \\
\hline$F V_{k}$ & Fixed cost of using transportation facility $k$ & $D I$ & Unbalanced distance spent \\
\hline
\end{tabular}

Variables

$x_{i j k} \quad$ One of the set of customers and storages $i$ in path $k$ is located after node $j$ and zero, otherwise

$y_{i} \quad$ One if storage $i$ is established and zero, otherwise

$z_{i j} \quad$ One if customer $i$ is assigned to storage $j$ and zero, otherwise

$U_{l k} \quad$ A dummy variable to remove sub tour

\section{Mathematical model}

$$
\begin{array}{ll}
\min \sum_{i \in I} F_{i} y_{i}+\sum_{k \in K} F V_{k} \sum_{i \in I} \sum_{j \in J} x_{i j k}+\sum_{i \in J} \sum_{j \in J} \sum_{k \in K} C_{i j} D_{i j} x_{i j k} & \\
\min D I=\operatorname{Max}\left(\sum_{i \in I} \sum_{j \in J} D_{i j} x_{i j k}\right)-\operatorname{Min}\left(\sum_{i \in I} \sum_{j \in J} D_{i j} x_{i j k}\right) & \forall k \in K
\end{array}
$$

subject to 
$\sum_{k \in K} \sum_{i \in I \cup J} x_{i j k}=1$

$\forall j \in J$

$\sum_{j \in I \cup J} x_{i j k}-\sum_{j \in I \cup J} x_{j i k}=0$

$\sum_{i \in I} \sum_{j \in J} x_{i j k} \leq 1$

$$
\sum_{j \in J} d_{j} \sum_{i \in I \cup J} x_{i j k} \leq Q
$$

$-z_{i j}+\sum_{u \in I \cup J}\left(x_{i u k}+x_{u j k}\right) \leq 1$

$\sum_{i \in I \cup J} \sum_{j \in I \cup J} t_{i j} x_{i j k}+\sum_{j \in J}\left(s_{j k}+w_{j k}\right) \sum_{j \in I \cup J} x_{i j k} \leq B$

$z_{i j} \leq y_{i}$

$U_{g k}-U_{j k}+N x_{g j k} \leq N-1$

$x_{i j k}, y_{i}, z_{i j} \in\{0,1\}$

$U_{l k} \geq 0$ $\forall k \in K, i \in I \cup J$

$\forall k \in K$

$\forall k \in K$

$\forall i \in I, j \in J, k \in K$

The first objective function minimizes transportation cost as well as fixed costs associated with storages and facilities. The second objective function minimizes unbalanced distance travelled. In other words, this objective function tries to setup a balance on distance travelled by all vehicles. According to Eq. (3), every customer uses only one vehicle while Eq. (4) states the sum of flows. Eq. (5) specifies that there is only one distance between two customers. Eq. (6) shows the capacity limitation for each vehicle. According to Eq. (7), a customer can only use a path when the path belongs to the same storage. Eq. (8) demonstrates total amount of traveling time. Eq. (9) specifies that we can assign a customer to a storage when the storage has already been established. Eq. (10) eliminates all sub-tours. Finally, Eq. (11) and Eq. (12) show the types of variables.

\section{Solution strategy}

The proposed study of this paper uses a hybrid of genetic and imperialist to solve the mathematical problem.

\subsection{Solution representation}

As is obvious, the efficiency and quality of a metaheuristics is entirely dependent on the representation of the solution. In addition, we have to be able to search the entire space using a good solution representation. Fig. 1 demonstrates the string, which is used for solution representation.

\begin{tabular}{|l|l|l|l|l|l|l|l|l|l|l|l|l|l|l|}
\hline 6 & 2 & 4 & 3 & 1 & 5 & 1 & 2 & 4 & 6 & 1 & 1 & 3 & 3 \\
\hline
\end{tabular}

Fig. 1. The representation of the solution 
Suppose there are $n$ customers, $m$ vehicles and $d$ candidates for storage. The solution representation contains $n+2 m$ components, consists of three parts. The first two parts are used for assignment of customers and sequencing and the third part is devoted for locating facilities. In fact, the first $n$ part shows the sequence of customers, the second $m$ part identifies the customers and finally, the third part represents the location of storage and where each vehicle should start its operations.

The Imperialist Competitive Algorithm (ICA) (Atashpaz-Gargari \& Lucas, 2007) begins by creating a set of candidate random solutions in the search space of the optimization process called the initial countries. Countries in this method are the counterpart of Chromosomes in genetic algorithms (GAs), which is an array with values of a candidate solution of optimization procedure and the objective function of the optimization problem detects the power of each country (Country $\left.=\left[p_{1}, p_{2}, p_{3}, \ldots, p_{N}\right]\right)$. Based on their power, some of the best initial countries become Imperialists and begin taking control of other colonies and form the initial Empires. Here the objective function is calculated as follows,

$$
\operatorname{Cost}_{i, n}=\frac{\left|f_{i, n}^{p}-f_{i, n}^{p, \text { best }}\right|}{f_{i, \text { total }}^{p, \text { max }}-f_{i, \text { total }}^{p, \min }}
$$

where $\operatorname{Cost}_{i, n}$ represents the normalized objective function $f_{i, n}^{p, \text { best }}, f_{i, \text { total }}^{p \text { max }}$ and $f_{i, \text { total }}^{p, \text { min }}$ represent the best, the maximum and the minimum objective functions, respectively. Next, the final normalized cost is calculated as follows,

$$
\text { Total Cost }_{n}=\sum_{i=1}^{r} \operatorname{Cost}_{i, n}
$$

In addition, the power of each empire is calculated as follows,

$$
p_{n}=\left|\frac{\text { Total Cost }_{n}}{\sum_{i=1}^{N_{\text {imp }}} \text { Total Cost }_{i}}\right|
$$

The number of colonies $\left(N C_{n}\right)$ is calculated as follows,

$$
N C_{n}=\operatorname{round}\left\{p_{n} \cdot N_{c o l}\right\} \text {, }
$$

where $N_{c o l}$ represents total number of colonies. Total power of empire (TP Emp $\left.p_{n}\right)$ is calculated as follows,

$$
\text { TP Emp } \text { Em }_{n}\left(\text { Total Cost }\left(\text { imperialist }_{n}\right)+\xi \operatorname{mean}\left\{\text { Total Cost }\left(\text { colonies of empire }{ }_{n}\right)\right\}\right)
$$

where $\xi$ is a positive number between zero and one. Fig. 2 shows the movement of colony towards Imperialist.

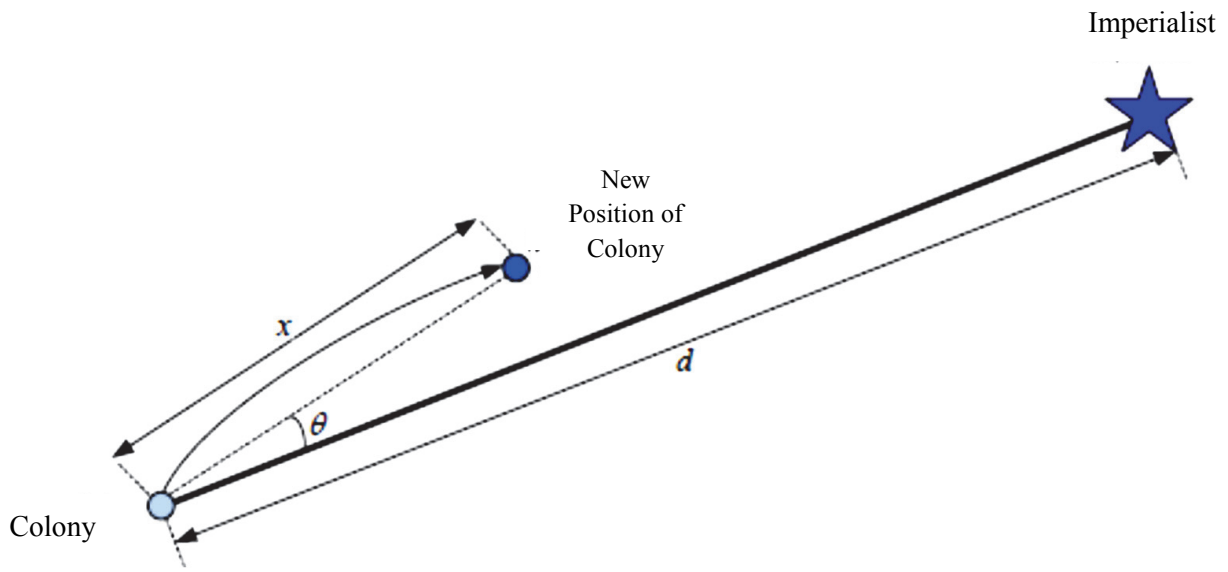

Fig. 2. The movement of colonies towards Imperialist 
As we can observe from Fig. $2, d$ represents the distance between colony and Imperialist, $x$ is a random number between zero and $\beta \times d$ where $\beta>1$. The movement is shown with $\theta$ and the rate of responses, which get close to Imperialist is demonstrated by $P_{A}$. To transfer data between the colonies crossover operator in genetic algorithm is used. For crossover, the proposed study uses single point and two point operations. Tournament selection method used to select the colonies. Also, $P_{c}$ represents a portion of the population chosen for crossover operations. In order to escape from local min, we use revolution. In competition, the power of weaker imperialist becomes less and more powerful imperialist become dominant. The likelihood of capturing each imperialist is calculated as follows,

$$
N T P E m p_{n}=\max \left\{T P E m p_{i}\right\}-T P E m p_{n}
$$

where NTP Emp $p_{n}$ represents total normalized power of imperialist $n, T P E m p_{n}$ states the power of imperialist $n$ and TP Emp $p_{i}$ denites the power of imperialist $i$. The possibility of capturing each imperialist is also calculated as follows,

$$
P_{p_{n}}=\left|\frac{N T P E m p_{n}}{\sum_{i=1}^{N_{i m p}} \text { TNTP Emp }}\right| .
$$

Finally, when there is only one imperialist, the algorithm terminates.

Deb et al. (2000) is the first who developed the idea of non-dominated sorting GA for multi-objective optimization known as NSGA-II method. Since we encounter with more than one optimal solution we have to handle multiple objective functions. Therefore, we show all efficient solutions in a set of Pareto optimal solutions. The following demonstrates the implementation of NSGAII originally developed by Parhizkari et al. (2013).

Step 1: Chromosome setting: The structure of the chromosome is designed based on a matrix.

Step 2: Evaluation: All generated solutions are assessed according to objective functions.

Step 3: Split solutions into two groups of dominated and non-dominated groups,

Step 4: Calculate design parameter: In this step, for each member of groups, calculate a parameter to compute the distance from the other members of group.

Step 5: Make a strategy to choose parents for the next generation,

Step 6: Execute two mutation and crossover operations to generate the new generation of solutions.

Step 7: Termination criteria: The proposed study of this paper terminates after some stopping criteria are met.

The results of the proposed method are compared with two other techniques of NSGA II and Pareto Archived Evolution Strategy (PAES) (Knowles \& Corne, 1999).

\section{The results}

In this section, we present details of the implementation of Imperialist and compares the method with two other methods, NSGA II and PAES. We have solved the problem for problems with different sizes. When there is more than one single objective function, authors must use Quality metric (QM), Mean ideal distance (MID), Spacing metric (SM) and Diversification metric (DM) to compare Pareto optimal solutions. The proposed study of this paper uses QM and SM to measure the quality of solutions. To measure QM, the contribution of each algorithm from Pareto solutions is demonstrated by this factor, in which simply measured by putting together the nondominated solutions detected by the algorithms and measuring the ratios between non-dominated solutions of each algorithm, where an algorithm with a higher value of the QM represents better performance. In addition, SM is calculated as follows, 


$$
S M=\frac{\sum_{i=1}^{n}\left|\bar{d}-d_{i}\right|}{(n-1) \bar{d}}
$$

where $d_{i}$ is the Euclidean distance between consecutive solutions in the calculated nondominated set of solutions and $\bar{d}$ is the average of these distances. Table 1 shows details of input parameters for the proposed method.

Table 1

The summary of the parameter used for the proposed study

\begin{tabular}{ccc}
\hline \multirow{2}{*}{ Factors } & \multicolumn{2}{c}{ Optimal real value } \\
\cline { 2 - 3 } & $\mathrm{S}$ & $\mathrm{L}$ \\
\hline $\mathrm{n}-\mathrm{Pop}$ & 193 & 300 \\
$\mathrm{~N}-\mathrm{imp}$ & 5 & 8 \\
$\mathrm{P}_{\mathrm{A}}$ & 0.54 & 0.64 \\
$\mathrm{P}_{\mathrm{C}}$ & 0.6 & 0.6 \\
$\mathrm{P}_{\mathrm{R}}$ & 0.12 & 0.32 \\
$\xi$ & 0.195 & 0.125 \\
$\beta$ & 1.8 & 2.15 \\
\hline
\end{tabular}

Tables (2-5) represent the performance of the proposed study for different size and number of distributions.

Table 2

The results of the survey for problems with sizes between 10 to 30 and distribution channels of 3 to 8

\begin{tabular}{|c|c|c|c|c|c|c|}
\hline \multirow{2}{*}{ Problem No. } & \multicolumn{3}{|c|}{ Quality Metric (QM) } & \multicolumn{3}{|c|}{ Spacing Metric (SM) } \\
\hline & NSGA-II & PAES & MOICA & NSGA-II & PAES & MOICA \\
\hline $10 \# 3$ & 0.235 & 0 & 0.765 & 0.827 & 0.625 & 0.741 \\
\hline $10 \# 4$ & 0.105 & 0 & 0.895 & 0.661 & 0.495 & 0.778 \\
\hline $15 \# 3$ & 0.250 & 0 & 0.750 & 0.791 & 0.788 & 0.920 \\
\hline $15 \# 4$ & 0 & 0 & 1 & 0.693 & 0.785 & 0.868 \\
\hline $15 \# 5$ & 0 & 0 & 1 & 0.571 & 1.092 & 0.634 \\
\hline $20 \# 3$ & 0 & 0 & 1 & 1.184 & 0.999 & 0.881 \\
\hline $20 \# 4$ & 0.235 & 0 & 0.765 & 0.778 & 1.257 & 0.973 \\
\hline $20 \# 5$ & 0.434 & 0 & 0.565 & 0.560 & 1.036 & 0.874 \\
\hline $20 \# 6$ & 0.347 & 0.217 & 0.434 & 0.733 & 1.031 & 0.924 \\
\hline $25 \# 3$ & 0.100 & 0 & 0.900 & 1.207 & 1.120 & 1.361 \\
\hline $25 \# 4$ & 0.272 & 0 & 0.727 & 1.001 & 0.878 & 1.287 \\
\hline $25 \# 5$ & 0.292 & 0 & 0.708 & 0.977 & 1.360 & 1.220 \\
\hline $25 \# 6$ & 0.190 & 0 & 0.809 & 0.940 & 0.977 & 1.481 \\
\hline $30 \# 3$ & 0.167 & 0.083 & 0.750 & 0.651 & 1.084 & 1.116 \\
\hline $30 \# 4$ & 0.059 & 0.294 & 0.647 & 1.059 & 1.322 & 0.810 \\
\hline $30 \# 5$ & 0 & 0 & 1 & 0.942 & 0.965 & 0.978 \\
\hline $30 \# 6$ & 0 & 0 & 1 & 0.670 & 0.916 & 0.980 \\
\hline $30 \# 7$ & 0.118 & 0 & 0.882 & 0.986 & 1.478 & 1.041 \\
\hline $30 \# 8$ & 0 & 0 & 1 & 0.586 & 0.911 & 0.642 \\
\hline
\end{tabular}

As we can observe from the results of Table 2, the proposed multi-objective ICA (MOICA) performs better than alternative methods; namely NSGA-II and PAES in terms of QM and SM.

Table 3

The results of the survey for problems with size of 40 and distribution channels between 3 to 10

\begin{tabular}{|c|c|c|c|c|c|c|}
\hline \multirow{2}{*}{ Problem No. } & \multicolumn{3}{|c|}{ Quality Metric (QM) } & \multicolumn{3}{|c|}{ Spacing Metric (SM) } \\
\hline & NSGA-II & PAES & MOICA & NSGA-II & PAES & MOICA \\
\hline $40 \# 3$ & 0.071 & 0 & 0.928 & 1.302 & 1.417 & 1.390 \\
\hline $40 \# 4$ & 0.3634 & 0.272 & 0.364 & 1.019 & 1.167 & 1.267 \\
\hline $40 \# 5$ & 0.357 & 0 & 0.642 & 1.628 & 1.295 & 1.649 \\
\hline $40 \# 6$ & 0.318 & 0.182 & 0.500 & 1.059 & 1.272 & 1.321 \\
\hline $40 \# 7$ & 0.370 & 0 & 0.630 & 0.984 & 1.374 & 1.372 \\
\hline $40 \# 8$ & 0.0416 & 0 & 0.958 & 1.037 & 1.296 & 0.935 \\
\hline $40 \# 9$ & 0.240 & 0 & 0.760 & 1.2678 & 1.049 & 1.410 \\
\hline $40 \# 10$ & 0.111 & 0.111 & 0.778 & 0.979 & 1.422 & 0.928 \\
\hline
\end{tabular}


The results of Table 3 and Table 4 indicate that MOICA in most cases perform better than the other alternative methods for medium size problems.

Table 4

The results of the survey for problems with size of 50 and distribution channels between 3 and 12

\begin{tabular}{|c|c|c|c|c|c|c|}
\hline \multirow{2}{*}{ Problem No. } & \multicolumn{3}{|c|}{ Quality Metric (QM) } & \multicolumn{3}{|c|}{ Spacing Metric (SM) } \\
\hline & NSGA-II & PAES & MOICA & NSGA-II & PAES & MOICA \\
\hline $50 \# 3$ & 0.434 & 0.086 & 0.478 & 1.339 & 1.461 & 1.479 \\
\hline $50 \# 4$ & 0.105 & 0 & 0.895 & 1.342 & 1.181 & 1.292 \\
\hline $50 \# 5$ & 0.238 & 0 & 0.762 & 1.465 & 1.548 & 1.466 \\
\hline $50 \# 6$ & 0 & 0 & 1 & 1.076 & 1.346 & 0.764 \\
\hline $50 \# 7$ & 0 & 0 & 1 & 1.042 & 1.249 & 1.446 \\
\hline $50 \# 8$ & 0 & 0 & 1 & 1.469 & 1.212 & 0.893 \\
\hline $50 \# 9$ & 0 & 0 & 1 & 0.753 & 1.202 & 1.432 \\
\hline $50 \# 10$ & 0 & 0.107 & 0.892 & 1.127 & 1.043 & 0.795 \\
\hline $50 \# 11$ & 0.160 & 0 & 0.840 & 0.952 & 0.998 & 0.863 \\
\hline $50 \# 12$ & 0 & 0 & 1 & 1.001 & 1.020 & 1.033 \\
\hline
\end{tabular}

Table 5

The results of the survey for problems with size of 70 and distribution channels between 3 and 16

\begin{tabular}{|c|c|c|c|c|c|c|}
\hline \multirow{2}{*}{ Problem No. } & \multicolumn{3}{|c|}{ Quality Metric (QM) } & \multicolumn{3}{|c|}{ Spacing Metric (SM) } \\
\hline & NSGA-II & PAES & MOICA & NSGA-II & PAES & MOICA \\
\hline $70 \# 3$ & 0 & 0 & 1 & 0.672 & 0.501 & 0.905 \\
\hline $70 \# 4$ & 0 & 0 & 1 & 0.517 & 1.299 & 0.811 \\
\hline $70 \# 5$ & 0 & 0 & 1 & 0.586 & 0.593 & 0.878 \\
\hline $70 \# 6$ & 0 & 0 & 1 & 0.737 & 0.402 & 0.752 \\
\hline $70 \# 7$ & 0.200 & 0 & 0.800 & 0.826 & 0.514 & 0.953 \\
\hline $70 \# 8$ & 0 & 0 & 1 & 0.495 & 1.032 & 0.427 \\
\hline $70 \# 9$ & 0 & 0.076 & 0.924 & 1.230 & 0.559 & 0.893 \\
\hline 70\#10 & 0 & 0 & 1 & 0.994 & 0.789 & 0.806 \\
\hline 70\#11 & 0 & 0 & 1 & 0.726 & 1.119 & 0.850 \\
\hline $70 \# 12$ & 0 & 0 & 1 & 0.632 & 0.904 & 0.608 \\
\hline $70 \# 13$ & 0 & 0 & 1 & 1.019 & 1.069 & 1.071 \\
\hline $70 \# 14$ & 0.352 & 0 & 0.647 & 0.721 & 1.024 & 0.721 \\
\hline $70 \# 15$ & 0.273 & 0 & 0.727 & 0.491 & 1.151 & 0.993 \\
\hline 70\#16 & 0 & 0 & 1 & 1.039 & 0.550 & 0.673 \\
\hline
\end{tabular}

Finally, Table 5 presents the results of the implementation of the proposed method when the sizes of the problems are relatively large. Once more, the proposed method has appeared to outperform the other alternative methods. Fig 3 shows the Pareto solutions for two different sizes of the problems solved.

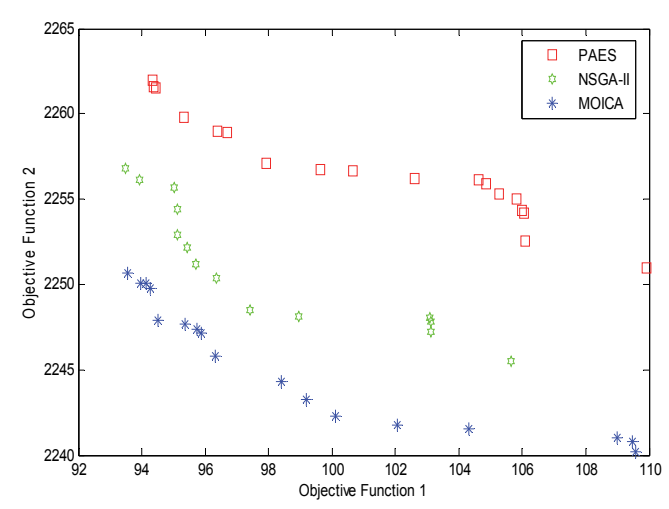

Pareto solutions for size $=30$ and 6 distribution channels

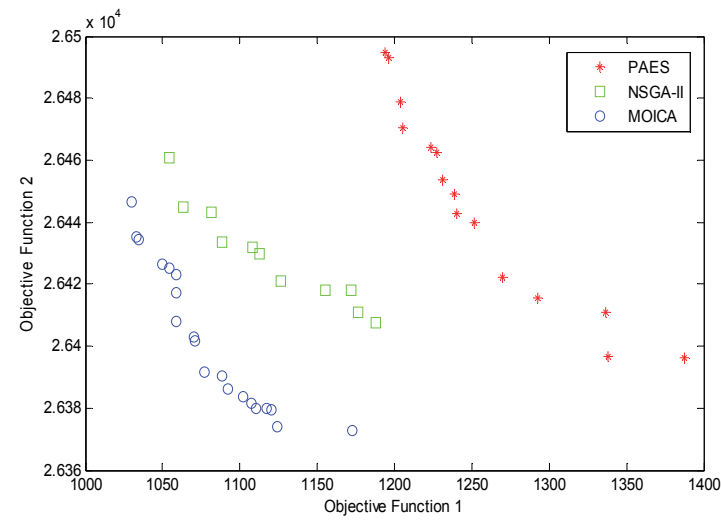

Pareto solutions for size $=70$ and 12 distribution channels

Fig. 3. The results of the Pareto solutions for small and large instances 


\section{Conclusion}

In this paper, we have presented a mathematical programming model to optimize the storage location and vehicle routing. The first objective function of the model minimized the total cost associated with the transportation and storage of rental fee, and the second objective function minimized the difference distance traveled by vehicles. We have used Imperialist Competitive Algorithm (ICA) to solve the resulted problem for different sizes of small, medium and large. The preliminary results have indicated that the proposed study has performed better than NSGA-II and PAES methods in terms of Quality metric and Spacing metric. As a future study, we may solve the problem by considering uncertainty in input parameters and we leave it for interested researchers as future studies.

\section{Acknowledgement}

The authors would like to thank the anonymous referees for constructive comments on earlier version of this paper.

\section{References}

Arvan, M., Tavakkoli-Moghaddam, R \& Abdollahi, M. (2015). Designing a bi-objective and multiproduct supply chain network for the supply of blood.Uncertain Supply Chain Management, 3(1), 5768.

Atashpaz-Gargari E, \& Lucas C. (2007). Imperialist competitive algorithm: an algorithm for optimization inspired by imperialistic competition. In: IEEE conference CEC.

Christofides, N., \& Eilon, S. (1969). An algorithm for the vehicle-dispatching problem. Operations Research, 20(3), 309-318.

Deb, K., Agrawal, S., Pratap, A., Meyarivan, T. (2000). A fast elitist non-dominated sorting genetic algorithm for multi-objective optimization: NSGA-II. In: proceedings of the parallel problem solving from nature VI (PPSN-VI) conference, 849-858.

Knowles, J., \& Corne, D. (1999). The pareto archived evolution strategy: A new baseline algorithm for pareto multiobjective optimisation. In Evolutionary Computation, 1999. CEC 99. Proceedings of the 1999 Congress on (Vol. 1). IEEE.

Parhizkari, M., Amiri, M., \& Mousakhani, M. (2013). A multiple criteria decision making technique for supplier selection and inventory management strategy: A case of multi-product and multi-supplier problem. Decision Science Letters, 2(3), 185-190.

Tavakkoli-Moghaddam, R., Saremi, A. R., \& Ziaee, M. S. (2006). A memetic algorithm for a vehicle routing problem with backhauls. Applied Mathematics and Computation, 181(2), 1049-1060.

Teimuory, E., Atoei, F., Mohammadi, E \& Amiri, A. (2013). A multi-objective reliable programming model for disruption in supply chain. Management Science Letters, 3(5), 1467-1478.

Webb, M. H. J. (1968). Cost functions in the location of depots for multiple-delivery journeys. Operations Research, 19(3), 311-320. 